\title{
Piomiositis en niños: experiencia en un hospital pediátrico de referencia en Argentina
}

\author{
M. Eugenia Pannunzio', M. Laura Praino', Carolina M. Carballo', Ana C. Medranda', \\ M. Nancy Orlando, Estefanía Josefina Biondi², Miryam S. Vázquez² y Eduardo L. López
}

\section{Pediatric pyomyositis: Experience in a Pediatric Reference Center in Argentina}

Background: Pyomyositis is the infection of skeletal muscle, a rare pathology in children. Aim. To describe the characteristics of pyomyositis in pediatric patients. Methods: Prospective analytical study of hospitalized children diagnosed with pyomyositis from May 2016 to April 2017 at the Ricardo Gutiérrez Children's Hospital, Buenos Aires, Argentina. Results: Twenty-one patients with pyomyositis were identified. Annual rate: 21.5/10,000 admissions (95\% CI 4.65-71.43). The median age was 5.4 years (range 1.25-11.6). The lower limbs were the most affected site. C-reactive protein (CRP) was elevated in all patients, with a mean of $124 \mathrm{mg} / \mathrm{L}$ (SD 96), being significantly higher in patients with bacteremia: 206 (DS 101) vs 98 (DS 81), p =0.02. Bacterial cultures were positive in 17/21 (80.9\%): 15 methicillin-resistant Staphylococcus aureus (MRSA), and 2 Streptococcus pyogenes. Blood cultures were positive in $5(23.8 \%)$. Conclusion: MRSA-community acquired is the predominant pathogen in our setting. In the selection of the appropriate empirical treatment, the local resistance pattern and the CRP value should be taken into account.

Keywords: Staphylococcal infections; pyomyositis; children; MRSA.

Palabras clave: Infecciones estafilocóccicas; piomiositis; niños; Staphylococcus aureus resistente a meticilina.

\section{Introducción}

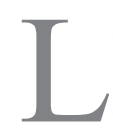

a piomiositis es la infección supurativa del músculo esquelético $^{1-3}$. Puede ser clasificada en primaria

o secundaria. El agente causal más frecuente es Staphylococcus aureus. Su incidencia ha aumentado aún en países de climas fríos a consecuencia de la emergencia de Staphylococcus aureus adquirido en la comunidad (SARM-AC) $)^{4}$ Las infecciones producidas por SARM productor de leucocidina de Panton Valentine se asocian a una evolución más tórpida ${ }^{5}$. Se describen factores predisponentes en 25 a $50 \%$ de los pacientes tales como: trauma previo, ejercicio intenso, desnutrición e inmunodeficiencias ${ }^{6}$. Las manifestaciones clínicas dependen del tiempo de evolución del cuadro y pueden clasificarse en tres estadios: inicial, supurativo y tardío ${ }^{1,2,6,7}$. No existen datos sobre la incidencia en edad pediátrica en nuestro país. El objetivo de nuestro trabajo fue describir la incidencia de piomiositis en niños, sus características clínicas y el manejo en la era de SARM-AC.

\section{Materiales y Métodos}

Se realizó un estudio prospectivo, analítico, en el que se incluyeron todos los pacientes entre 0 y 18 años de edad internados con diagnóstico de piomiositis en un hospital pediátrico de alta complejidad entre mayo de 2016 y abril de 2017. Se diseñó una ficha de recolección de datos que incluyó: edad, sexo, co-morbilidad, antecedentes personales o familiares de infecciones cutáneas, factores de riesgo como lesiones de piel, traumatismos o ejercicio intenso, datos de presentación y evolución del cuadro actual.

Se utilizaron variables estadísticas de distribución y posición que fueron procesadas con el programa STATA versión 13.

\section{Definiciones}

Piomiositis: Infección supurativa del músculo esquelético. El diagnóstico fue realizado, según lo referido en la bibliografía, en base a la presentación clínica (dolor, tumefacción, impotencia funcional y fiebre), los estudios de imágenes y/o la exploración quirúrgica ${ }^{4,8}$. Se clasifica como primaria si se produce por diseminación hematógena sin un foco o puerta de entrada y secundaria cuando se produce por infección de un sitio adyacente $\mathrm{e}^{6,3}$.

Microbiología: Los materiales purulentos se cultivaron en medios sólidos y líquidos. En la mayoría de los casos habían sido inoculados en botellas de hemocultivos; para éstos y para los hemocultivos se utilizó el sistema Bact-alert (bioMérieux, Marcy, L’Étoile, Francia). La identificación se realizó mediante metodología fenotípica
'Hospital de Niños Ricardo Gutiérrez. Hospital asociado a la Facultad de Medicina de la Universidad de Buenos Aires. Servicio de Infectología Pediátrica. Buenos Aires, Argentina. ${ }^{2}$ Hospital de Niños Ricardo Gutiérrez. Servicio de Microbiología. Buenos Aires, Argentina.

Ningún autor presenta conflictos de interés.

No se cuenta con fuentes de financiación externa.

Recibido: 25 de julio de 2018 Aceptado:

Correspondencia a: María Eugenia Pannunzio eugeniapannunzio@gmail.com 
convencional y/o espectrometría de masas Vitek MS ${ }^{\mathrm{TM}}$ (bioMérieux). La sensibilidad antimicrobiana se estudió por el método de difusión en agar (técnica de KirbyBauer) según los lineamientos del Clinical and Laboratory Standards Institute (CLSI). La concentración inhibitoria mínima (CIM) a vancomicina se ensayó por Vitek2 (bioMérieux, Marcy, L'Étoile, Francia), de acuerdo a las indicaciones del fabricante.

$S A R M-A C$ : Se define como infecciones adquiridas en la comunidad aquellas que presentaron el inicio de sus manifestaciones clínicas antes de ingresar al hospital o durante las primeras $48 \mathrm{~h}$ y en pacientes que no presentaban patologías que condicionaran la concurrencia frecuente a un centro de salud ni internaciones recientes.

Tabla 1. Manifestaciones clínicas al ingreso de 21 pacientes con piomiositis

$\begin{array}{lcc}\text { Signos y síntomas } & \text { N } & \% \\ \text { Fiebre } & 21 & 100 \\ \text { Dolor local } & 17 & 81 \\ \text { Tumefacción } & 12 & 57 \\ \text { Impotencia funcional } & 11 & 52 \\ \text { Calor local } & 8 & 38 \\ \text { Eritema } & 5 & 24\end{array}$

Figura 1. Resonancia magnética, secuencia T1 de miembros inferiores en paciente con piomiositis bilateral de muslos. Corte coronal que evidencia hiperintensidad en aductores (flechas).

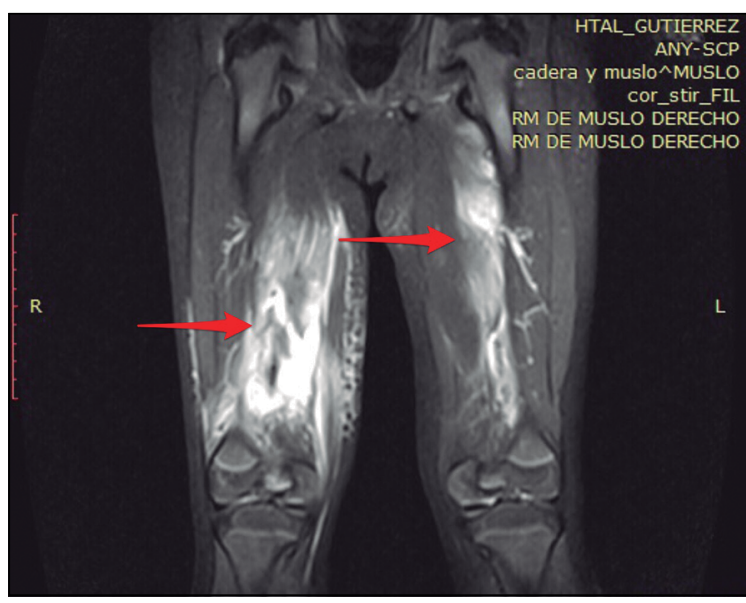

Figura 2. Aislamiento microbiológico.

\section{Resultados}

Durante el período de estudio se incluyeron 21 pacientes con diagnóstico de piomiositis con una tasa de hospitalización de 21,5/10.000/año (IC 95\% 4,65- 71,43). La mediana de edad fue de 5 años (rango 1,25-11,5), con una relación mujer: varón de 1,6: 1 .

El 90,4\% (19/21) presentaba algún factor predisponente: traumatismo cerrado $9 / 21(42,2 \%)$, antecedente personal o familiar de forunculosis $8 / 21(38 \%)$, infección de piel y tejidos blandos $2 / 21(9,8 \%)$. No se encontraron en esta serie pacientes inmunosuprimidos.

La mediana de días al diagnóstico fue de tres (rango 1 a 14).

Las zonas más afectadas fueron los miembros inferiores en nueve pacientes $(42,8 \%)$ seguido por infecciones en el tronco seis $(28,5 \%)$, miembros superiores cinco $(23,8 \%)$ y multifocal uno $(4,9 \%)$

La forma de presentación más frecuente fue la piomiositis abscedada en 19/21 (90\%). Sólo dos pacientes llegaron a la internación con un cuadro clínico de sepsis con impactos múltiples.

Los signos y síntomas de presentación se detallan en la Tabla 1. El 62\% (13/21) fueron piomiositis primarias, mientras que en ocho casos la infección muscular fue secundaria a un foco adyacente (siete infecciones cutáneas y un absceso renal).

$\mathrm{Al}$ ingreso, $85,7 \%$ de los pacientes mostró leucocitosis con una mediana de leucocitos de $21.600 / \mathrm{ml}$ (rango 6.100 a 47.200). La PCR estuvo elevada en todos los pacientes con una media de $124 \mathrm{mg} / \mathrm{L}$ (DS 96), y fue más elevada en los pacientes que tuvieron hemocultivos positivos, 206 (DS 101) vs 98 (DS 81): Wilcoxon S-R Test, $\mathrm{p}=0,02$.

De las 20 ecografías realizadas, 18 mostraron el diagnóstico $(90 \%)$, mientras que las dos restantes fueron normales y se llegó al diagnóstico por resonancia magnética (RM) (Figura 1). Un paciente se presentó con síndrome compartimental y el diagnóstico fue realizado en el acto quirúrgico. Tres niños presentaron enfermedad complicada: dos con sepsis e impactos a distancia y uno con síndrome compartimental y fascitis necrosante.

Se obtuvo rescate microbiológico en 17 pacientes (Figura 2). En 16 casos desde el material purulento de músculo (obtenido por punción o cirugía); en cinco de hemocultivos, mientras que cuatro pacientes tuvieron ambos materiales positivos. Staphylococcus aureus fue el principal agente etiológico (15/17). Todos los aislados de $S$. aureus fueron resistentes a meticilina y sensibles a cotrimoxazol (SXT), rifampicina, minociclina y ciprofloxacina. Tres resultaron resistentes a clindamicina y dos a gentamicina. $\mathrm{La} \mathrm{CIM}_{90}$ a vancomicina fue de $1 \mu \mathrm{g} /$ $\mathrm{ml}$. En dos casos, se rescató Streptococcus pyogenes en material purulento sin bacteriemia acompañante.

El tratamiento empírico inicial fue dirigido frente a 
bacterias grampositivas y en todos se inició por vía endovenosa. El antimicrobiano más utilizado fue clindamicina (40 mg/kg/día) en 18/21 pacientes (monoterapia en cinco, combinada con vancomicina en nueve, con ceftriaxona en tres y uno con aminoglucósido). Dos pacientes recibieron monoterapia con vancomicina $(60 \mathrm{mg} / \mathrm{kg} /$ día $)$ y uno con SXT $(10 \mathrm{mg} / \mathrm{kg} /$ día de trimetoprim). La indicación de vancomicina como tratamiento empírico fue realizada en pacientes con mayor gravedad del cuadro clínico. Sólo un paciente recibió tratamiento inadecuado ya que fue tratado con clindamicina y se trataba de un SARM-AC resistente a este fármaco. La duración del tratamiento endovenoso tuvo una mediana de 17 días (rango 7-41). El 81\% de los pacientes requirió drenaje quirúrgico. Tres pacientes requirieron internación en Unidad de Cuidados Intensivos, de quienes sólo uno requirió inotrópicos y asistencia respiratoria mecánica.

El 81\%,17/21 pacientes, completó su tratamiento vía oral con SXT sin presentar recaídas ni efectos adversos a fármacos. La recuperación fue completa en 20/21 mientras que el niño que ingresó con síndrome compartimental por SARM presentó secuelas motoras. Ninguno falleció.

\section{Discusión}

La piomiositis es una infección bacteriana del músculo esquelético que puede resultar en la formación de abscesos $^{1,2,9}$. Es una entidad habitual en áreas tropicales, donde fue inicialmente descrita por Scriba en $1885^{4,9}$. La incidencia en países con clima templado parece estar en aumento, con una tasa de hospitalización de 3,3/10.000 $\mathrm{y}$ es posible que se relacione con la emergencia de SARM-AC ${ }^{9}$. En nuestro estudio observamos una tasa de hospitalización de 21/10.000, seis veces mayor a la reportada. En nuestro país la incidencia de SARM-AC ha mostrado un aumento en los últimos años siendo 68\% en un trabajo realizado en nuestro servicio entre los años 2010 y $2011^{10}$.

La edad de presentación más frecuente fue entre 2,9 y 6,8 años (RIQ), mediana de 5 años, en coincidencia con el resto de la literatura médica ${ }^{1,6,9}$. Es llamativo que en nuestra serie de casos la incidencia fue mayor en mujeres que en varones, a diferencia de lo reportado por estudios previos $^{6,11}$.

En regiones con menor prevalencia de piomiositis, así como en la población adulta, se relaciona con más frecuencia al inmunocompromiso ${ }^{2,11}$. En contraste, los niños internados en nuestro hospital eran todos inmunocompetentes poniendo de relieve la importancia de la sospecha clínica, aun sin dicho factor de riesgo.

Se postula que la injuria muscular previa por traumatismo o el ejercicio intenso serían factores predisponentes para el desarrollo de piomiositis primaria ${ }^{1-3,6}$. El 43\% de nuestra serie, presentó el antecedente de injuria muscular. Las piomiositis secundarias se producen a partir de infecciones adyacentes como ser celulitis, osteomielitis, absceso renal ${ }^{5}$. La mitad de nuestros casos, presentó un foco contiguo. Siete pacientes iniciaron su cuadro a punto de partida de una celulitis, dos fueron secundarias a osteomielitis y uno desarrolló una piomiositis del psoas como extensión de un absceso renal.

En nuestro trabajo, la mayoría de los pacientes (19/21) se presentaron como piomiositis abscedada, al igual que lo descrito en la literatura científica ${ }^{1,7}$. Sólo dos pacientes llegaron a la internación con un cuadro de sepsis.

Las manifestaciones clínicas dependen de la localización y del estadio de evolución en que se detecta, desde un cuadro de dolor muscular, con o sin impotencia funcional y fiebre, hasta un estado séptico. La lesión es habitualmente dolorosa a la palpación, pero el eritema y edema cutáneo son infrecuentes, excepto cuando hay compromiso del tejido celular subcutáneo ${ }^{1,6}$. Los síntomas más frecuentes en nuestros pacientes fueron fiebre, dolor local y tumefacción. Puede comprometer cualquier músculo o grupo muscular, aunque predomina su localización en las extremidades inferiores y cintura pélvica, pudiendo ser multifocal en hasta $40 \%$ de los $\operatorname{casos}^{2,4,7}$. En nuestra serie, $43 \%$ de los pacientes se presentó con compromiso en miembros inferiores, seguido en frecuencia por la localización en tronco $(28,5 \%)$. Sin embargo, la presentación muscular multifocal fue menos frecuente que lo descrito $(4,7 \%)$.

El laboratorio es poco específico para realizar el diagnóstico ${ }^{2}$. El 85,7\% presentó leucocitosis. Todos los pacientes presentaron aumento de la PCR, que fue significativamente más elevada en los pacientes con bacteriemia $(p=0,02)$.

El agente etiológico aislado con más frecuencia, como ocurrió en nuestros pacientes, es $S$. aureus, con una frecuencia de $95 \%$ en climas tropicales y $70 \%$ en climas templados, independientemente del estado inmunológico del paciente ${ }^{6}$. Streptococcus pyogenes se describe como el segundo agente causal ${ }^{2,7}$. En pacientes con compromiso inmunológico pueden aislarse Escherichia coli, Yersinia enterocolitica, Klebsiella spp, Salmonella spp, especies anaerobias estrictas, hongos o micobacterias ${ }^{6}$. En la bibliografía se describe que aproximadamente un tercio de los pacientes presenta bacteriemia mientras que en esta serie se evidenció en $23,8 \%{ }^{6}$. El aislamiento microbiológico es más frecuente en el material de drenaje, lo que coincide con nuestros resultados ${ }^{4}$.

En cuanto a los métodos diagnósticos, la ecografía se reconoce como estudio inicial, dada su mayor disponibilidad y rapidez, pero con la limitación de no detectar la piomiositis en los estadios iniciales y de ser un examen operador dependiente ${ }^{2,7}$. La RM se considera el mejor método para el diagnóstico temprano, dado que es capaz 
de detectar pequeños cambios inflamatorios en tejidos blandos, además de ayudar en el diagnóstico diferencial con otras patologías ${ }^{2,3,5,8}$. La ecografía logró identificar a $90 \%$ de las piomiositis en nuestro trabajo (es de destacar que todas se encontraban abscedadas), mientras que en el $10 \%$ restante el diagnóstico se hizo mediante RM.

El tratamiento empírico inicial depende principalmente de la epidemiología y patrones de resistencia locales ${ }^{5,12}$. En lugares de alta prevalencia de SARM-AC, éste agente debe ser considerado en el esquema empírico inicial. Si los resultados microbiológicos informan $S$. aureus sensible a meticilina, el tratamiento debe realizarse con cefalosporinas de primera generación o penicilinas anti estafilocóccicas $^{4,8}$. En nuestro hospital, en el período 2010 2011 se han descrito tasas de SARM-AC en la comunidad de $68 \%$, con resistencia a clindamicina $8,5 \%$ y a SXT menor a $1 \%{ }^{10,12}$. En esta serie de pacientes, la resistencia a clindamicina ascendió a $20 \%$. Dado que se trata de un número pequeño de pacientes, no puede realizarse una recomendación respecto al uso de este antimicrobiano, pero debe estarse alerta sobre un posible aumento de la resistencia, lo que limitaría su uso en el esquema empírico. SXT fue el fármaco más utilizado en el tratamiento de continuación (oral), siendo eficaz y bien tolerado.

La formación de un absceso exige un apropiado drenaje y desbridamiento de los tejidos afectados, fundamental para lograr el éxito terapéutico ${ }^{3,5}$. El mismo puede ser a través del drenaje percutáneo guiado bajo ecografía o $\mathrm{TAC}^{7}$. El 81\% de nuestros pacientes requirió drenaje quirúrgico.

En cuanto a la duración del tratamiento antimicrobiano endovenoso, el mismo debe continuarse hasta observar mejoría clínica e imagenológica, para luego continuar tratamiento por vía oral ${ }^{6,9}$. En pacientes sin complicaciones, dos a seis semanas de terapia parecen ser suficientes ${ }^{2,6,11}$. Nuestros pacientes, al igual que lo reportado, requirieron una mediana de 17 días (rango 7 a 41).

El tratamiento adecuado lleva a la recuperación completa en la mayoría de los casos, pero se han descrito complicaciones locales o a distancia, e incluso una mortalidad de $10 \%$ en el período agudo ${ }^{6,11}$. En nuestra serie no hubo fallecidos; sin embargo, el niño que se presentó con un síndrome compartimental presentó una lesión nerviosa con limitación grave de la motilidad distal de la mano.

\section{Conclusiones}

La piomiositis presenta en nuestro medio una incidencia mayor a la de países de clima frío. Puede presentarse en niños inmunocompetentes, siendo frecuente el antecedente de injuria muscular o infecciones cutáneas. Se requiere un alto índice de sospecha para no demorar el diagnóstico. Staphylococcus aureus es el agente causal más frecuente. Los patrones de resistencia local deben ser monitoreados para guiar el esquema empírico. El valor de PCR al ingreso podría indicar mayor riesgo de bacteriemia. La ecografía resulta un adecuado método diagnóstico, especialmente en piomiositis abscedada. La antibioticoterapia endovenosa y el drenaje quirúrgico son los pilares del tratamiento, con buen pronóstico en la mayoría de los casos.

\section{Resumen}

Introducción: La piomiositis es la infección del músculo esquelético, entidad poco frecuente en pediatría. Objetivo: Describir las características de 21 niños con piomiositis. Métodos: Estudio prospectivo-analítico de niños ingresados con diagnóstico de piomiositis entre mayo de 2016 y abril de 2017 en el Hospital de Niños Ricardo Gutiérrez, Buenos Aires, Argentina. Resultados: Tasa de hospitalización de 21,5/10.000 admisiones (IC 95\% $4,65-71,43$ ). La mediana de edad fue de 5,4 años (rango 1,25-11,6). El 90,4\% presentaba algún factor predisponente. La localización más frecuente fue en miembros inferiores. La proteína $\mathrm{C}$ reactiva (PCR) estuvo elevada en todos los pacientes, con una media de $124 \mathrm{mg} / \mathrm{L}$ (DS 96), siendo significativamente más elevada en los pacientes que tuvieron hemocultivos positivos 206 (DS 101) vs 98 (DS 81$),(p=0,02)$. Se obtuvo rescate microbiológico en 17 pacientes (80,9\%): Staphylococcus aureus resistente a meticilina (SARM) (n: 15) y Streptococcus pyogenes (n: 2). Se presentó con bacteriemia $23,8 \%$ de los pacientes. El $81 \%$ requirió drenaje quirúrgico. Conclusión: Staphylococcus aureus RM adquirido en la comunidad (SARMAC) es el patógeno predominante. En la selección del tratamiento empírico adecuado debería tenerse en cuenta: el patrón de resistencia local y el valor de PCR. 


\section{Referencias bibliográficas}

1.- Chiedozi L C. Pyomyositis. Review of 205 cases in 112 patients. Am J Surg 1979; 137(2): 255-9. PMID: 426186

2.- Mitsionis G I, Manoudis G N, Lykissas M G, Sionti I, Motsis E, Georgoulis A D, et al. Pyomyositis in children: early diagnosis and treatment. J Pediatr Surg 2009; 44 (11): 2173-8. doi: 10.1016/j.jpedsurg.2009.02.053.

3.- Comegna L, Guidone P I, Prezioso G, Franchini S, Petrosino M I, Di Filippo P, et al. Pyomyositis is not only a tropical pathology: a case series. J Med Case Rep 2016; 10: 372. doi: 10.1186/s13256-016-1158-2.

4.- Pannaraj P S, Hulten K G, González B E, Mason E O Jr, Kaplan S L. Infective pyomyositis and myositis in children in the era of community-acquired, methicillin-resistant Staphylococcus aureus infection. Clin Infect Dis 2006; 43 (8): 953-60. doi: 10.1086/50763.
5.- Kaplan S L. Implications of methicillinresistant Staphylococcus aureus as a community-acquired pathogen in pediatric patients. Infect Dis Clin North Am 2005; 19(3): 747-57. doi: 10.1016/j.idc.2005.05.011.

6.- Cavagnaro F, Rodríguez J, Arancibia M E, Walker B, Espinoza A. Piomiositis en niños. Reporte de dos casos. Rev Chilena Infectol 2013; 30: 81-5. doi: 10.4067/S071610182013000100014.

7.- Unnikrishnan P N, Perry D C, George H, Bassi R, Bruce C E. Tropical primary pyomyositis in children of the UK: an emerging medical challenge. Int Orthop 2010; 34 (1): 109-13. doi: 10.1007/s00264-009-0765-6.

8.- Stevens D L, Bisno A L, Chambers H F, Dellinger E P, Goldstein E J, Gorbach S L, et al. Practice guidelines for the diagnosis and management of skin and soft tissue infections: 2014 Update by the Infectious Diseases Society of America. Clin Infect Dis 2014; 59 (2): e10-e52. doi: 10.1093/cid/ciu296.

9.- Christin L, Sarosi G A. Pyomyositis in North America: case reports and review. Clin Infect Dis. 1992; 15: 668-77. PMID: 1420680.

10.- Praino M L, Neyro S, Vázquez M, Procopio A, Alvarez M S, Juárez X, et al. Infecciones por Staphylococcus aureus en el Hospital de Niños "Dr Ricardo Gutiérrez". Poster presentado en el "III Congreso de Infectología Pediátrica y Vacunas" CABA, Argentina. Abril 2012.

11.- Bickels J, Ben-Sira L, Kessler A, Weintroub S. Primary pyomyositis. J Bone Joint Surg 2002; 84-A: 2277-86. PMID: 12473721.

12.- Bermejo V, Spadaccini L, Elbert G, Duarte A, Erbin M, Cahn P. Prevalencia de Staphylococcus aureus resistente a meticilina en infecciones de piel y partes blandas en pacientes ambulatorios. Medicina (Buenos Aires) 2012; 72: 283-6. http://www.scielo.org. ar/pdf/medba/v72n4/v72n4a02.pdf. 